\title{
Non-Born-Oppenheimer variational calculation of the ground-state vibrational spectrum of $\mathrm{LiH}^{+}$
}

\author{
Sergiy Bubin ${ }^{\text {a) }}$ \\ Department of Physics, University of Arizona, Tucson, Arizona 85721 \\ and Department of Chemistry, University of Arizona, Tucson, Arizona 85721 \\ Ludwik Adamowicz \\ Department of Chemistry, University of Arizona, Tucson, Arizona 85721 \\ and Department of Physics, University of Arizona, Tucson, Arizona 85721
}

(Received 2 June 2006; accepted 6 July 2006; published online 11 August 2006)

\begin{abstract}
Very accurate, rigorous, variational, non-Born-Oppenheimer (non-BO) calculations have been performed for the fully symmetric, bound states of the $\mathrm{LiH}^{+}$ion. These states correspond to the ground and excited vibrational states of $\mathrm{LiH}^{+}$in the ground ${ }^{2} \Sigma^{+}$electronic state. The non-BO wave functions of the states have been expanded in terms of spherical $N$-particle explicitly correlated Gaussian functions multiplied by even powers of the internuclear distance and 5600 Gaussians were used for each state. The calculations that, to our knowledge, are the most accurate ever performed for a diatomic system with three electrons have yielded six bound states. Average interparticle distances and nucleus-nucleus correlation function plots are presented. (C) 2006 American Institute of Physics. [DOI: 10.1063/1.2244563]
\end{abstract}

\section{INTRODUCTION}

Our previous works concerning non-Born-Oppenheimer (non-Bo) variational calculation of the vibrational spectrum of the $\mathrm{H}_{2}$ molecule ${ }^{1,2}$ where we used 3000 and 5000 explicitly correlated Gaussian functions (ECGFs), respectively, for each state yielded a very similar accuracy for all 15 vibrational transition energies as that achieved a decade ago by Wolniewicz. ${ }^{3}$ Since, unlike Wolniewicz's, our approach is not restricted to two-electron systems, we decided to undertake a series of rigorous, variational, non-BO calculations of all vibrational states of a three-electron system in its ground electronic state. The vibrational spectrum of the system we have chosen, the $\mathrm{LiH}^{+}$ion, has been studied before using methods based on the $\mathrm{BO}$ approximation. ${ }^{4,5}$ The present calculations on $\mathrm{LiH}^{+}$are not only the first non-BO calculations on this system but also the first non-BO calculations of the vibrational spectrum ever performed for a three-electron diatomic molecule.

In a non-BO calculation one does not assume the separability of the electronic and nuclear motions and the wave function depends explicitly on the coordinates of all particles involved in the system. In such a situation the correlation effects, which in the BO calculations are restricted to electrons, also appear in the relative nucleus-nucleus motion, as well as in the relative motion involving the nuclei and the electrons. For this reason, non-BO calculations are significantly more complicated and time consuming than $\mathrm{BO}$ calculations. Moreover, if a high precision of non-BO calculations is required the basis functions used have to explicitly depend on the distances between particles (this is why explicitly correlated Gaussians have been used in the present work), and not only long expansions of the wave function in

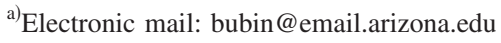

terms of basis functions must be used but also linear and nonlinear parameters of the basis function have to be extensively optimized.

The non-BO calculations presented in this work were performed using the method we have been developing in the last few years. For a description of the method, we refer the reader to our recent reviews. ${ }^{6,7}$ In the next section we will briefly describe the method and its application to calculate the vibrational states of $\mathrm{LiH}^{+}$. We will also briefly describe the procedure used to optimize the linear and nonlinear parameters of the wave functions. It should be noted that the term "vibrational states" can only approximately describe states with zero total angular momentum considered in this work. This is because if the BO approximation is not assumed, the vibrational motion is coupled with the electronic motion and, strictly speaking, the vibrational quantum number is not a good quantum number.

\section{THE NON-BO APPROACH}

In the non-BO calculation we start with the nonrelativistic $N$-particle Hamiltonian in the laboratory Cartesian coordinate system. The particles are electrons and nuclei:

$$
\hat{H}_{\mathrm{tot}}=-\sum_{i=1}^{N} \frac{1}{2 M_{i}} \nabla_{\mathbf{R}_{i}}^{2}+\sum_{i<j=1}^{N} \frac{Q_{i} Q_{j}}{R_{i j}} .
$$

The masses, charges, and positions of the particles in (1) are denoted as $M_{i}, Q_{i}$, and $\mathbf{R}_{i}$, respectively. Next we rigorously separate out from $\hat{H}_{\text {tot }}$ the motion of the center of mass. This is accomplished by a coordinate transformation. The new coordinate system comprises three laboratory coordinates of the center of mass and $3 N-3$ internal Cartesian coordinates. The center of the internal coordinate system is placed at one of the particles (usually the heaviest one) called the reference 
TABLE I. Non-BO total energies of $\mathrm{LiH}^{+}$vibrational states obtained with $3200,3600,4000,4400,4800,5200$, and 5600 basis functions. All values are in a.u.

\begin{tabular}{cccccccc}
\hline \hline$v$ & 3200 & 3600 & 4000 & 4400 & 4800 & 5200 & 5600 \\
\hline 0 & -7.78324642 & -7.78324660 & -7.78324672 & -7.78324679 & -7.78324684 & -7.78324688 & -7.78324691 \\
1 & -7.78162705 & -7.78162757 & -7.78162795 & -7.78162820 & -7.78162845 & -7.78162866 & -7.78162885 \\
2 & -7.78043317 & -7.78043426 & -7.78043497 & -7.78043546 & -7.78043580 & -7.78043603 & -7.78043619 \\
3 & -7.77965589 & -7.77965802 & -7.77965930 & -7.77966016 & -7.77966073 & -7.77966113 & -7.77966140 \\
4 & -7.77924363 & -7.77924715 & -7.77924915 & -7.77925034 & -7.77925107 & -7.77925156 & -7.77925191 \\
5 & -7.77903864 & -7.77907542 & -7.77908456 & -7.77908792 & -7.77908950 & -7.77909042 & -7.77909093 \\
\hline \hline
\end{tabular}

particle and the internal coordinate axes are made parallel to the axes of the laboratory coordinate frame. In the internal coordinate system the particles other than the reference particle are referred to the reference particle using the position vectors $\mathbf{r}_{i}, i=1, \ldots, n$, where $n=N-1$. After the coordinate transformation the laboratory Hamiltonian $\hat{H}_{\text {tot }}$ separates into the Hamiltonian describing the kinetic energy of the centerof-mass motion and the following internal Hamiltonian $\hat{H}$ :

$$
\begin{aligned}
\hat{H}= & -\frac{1}{2}\left(\sum_{i=1}^{n} \frac{1}{m_{i}} \nabla_{\mathbf{r}_{i}}^{2}+\sum_{i \neq j=1}^{n} \frac{1}{M_{1}} \nabla_{\mathbf{r}_{i}}^{\prime} \nabla_{\mathbf{r}_{j}}\right) \\
& +\sum_{i=1}^{n} \frac{q_{0} q_{i}}{r_{i}}+\sum_{j>i}^{n} \frac{q_{i} q_{j}}{r_{i j}} .
\end{aligned}
$$

In (2) $q_{0}=Q_{1}$ is the charge of the reference particle, $q_{i}=Q_{i+1}(i=1, \ldots, n)$ are the charges of the other $n$ particles, $r_{i j}=\left|\mathbf{r}_{j}-\mathbf{r}_{i}\right|$, and the prime ( $\left.{ }^{\prime}\right)$ is used to indicate vectormatrix transposition. The internal Hamiltonian (2) describes $n$ pseudoparticles with charges $q_{i}$ and reduced masses $m_{i}$ $=M_{1} M_{i+1} /\left(M_{1}+M_{i+1}\right)$ moving in the spherical potential of charge $q_{0}$. In this work, particle 1 , the reference particle, was the Li nucleus, particle 2 was the proton, and particles 3,4 , and 5 were the electrons.

The key element of the non-BO calculation is the selection of the basis set for the expansion of the wave function. Since the internal Hamiltonian $\hat{H}$ is spherically symmetric (i.e., isotropic with respect to any rotation about the center of the internal coordinate system), the basis functions for describing states corresponding to zero angular momentum (i.e., the vibrational states) have to be spherically symmetric as well. In our works concerning non-BO calculations on light diatomic molecular systems (see, for example, Refs. 1, 2, and 8 and references in Refs. 6 and 7), we have shown that the explicitly correlated Gaussians (ECGs) involving functions with preexponential multipliers consisting of the internuclear distance $r_{1}$ raised to a non-negative even power $m_{k}$,

$$
\phi_{k}=r_{1}^{m_{k}} \exp \left[-\mathbf{r}^{\prime}\left(A_{k} \otimes I_{3}\right) \mathbf{r}\right]=r_{1}^{m_{k}} \exp \left[-\mathbf{r}^{\prime} \bar{A}_{k} \mathbf{r}\right] \text {, }
$$

are very effective in describing such states. In (3) $A_{k}$ is a symmetric, positive definite, $n \times n$ matrix, and $\bar{A}_{k}$ is a Kronecker product of $A_{k}$ and $I_{3}, \bar{A}_{k}=A_{k} \otimes I_{3}$, where $I_{3}$ is the $3 \times 3$ identity matrix.

In accordance with the Pauli principle, the basis functions (3) must have the proper permutational symmetry so that when the spatial and spin parts are combined the result- ing function is antisymmetric with respect to interchanging the electron labels. In our calculations the antisymmetrization was implemented using the standard approach based on Young operators, as described in Ref. 9.

In the non-BO calculations we use the variational method and we minimize the energy functional (the Rayleigh quotient)

$$
E\left(\left\{c_{k}\right\},\left\{m_{k}\right\},\left\{A_{k}\right\}\right)=\min \frac{c^{\prime} H\left(\left\{m_{k}\right\},\left\{A_{k}\right\}\right) c}{c^{\prime} S\left(\left\{m_{k}\right\},\left\{A_{k}\right\}\right) c}
$$

with respect to the linear expansion coefficients $c_{k}$, the basis function exponential parameters $\left\{A_{k}\right\}$, and the preexponential powers $\left\{m_{k}\right\}$. The optimization is done using an algorithm based on analytical derivatives of the functional (4) with respect to the exponential parameters. The optimization of the basis set was performed separately for each state and the basis was grown from a small size to a set of 5600 functions. We believe that with this many functions the total energies are converged to about seventh decimal figure. The convergence is certainly better for the lower lying states than for the higher states due to increasingly more complicated structure (larger number of nodes) in the wave functions of the higher vibrational states. The preexponential powers $\left\{m_{k}\right\}$ in our calculations ranged from 0 to 250 and they were partially optimized for each state. More details on the Hamiltonian transformation, proper symmetrization of the basis functions, and selection of nonlinear parameters for calculations with ECGFs can be found in Refs. 6 and 7. The masses of the

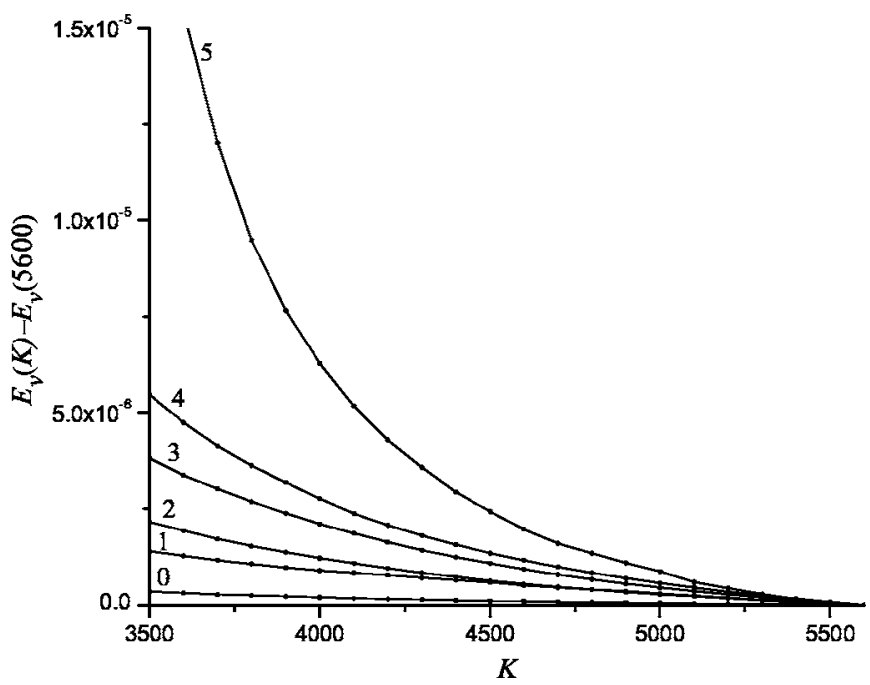

FIG. 1. Convergence of the total energy, $E_{v}$, for states with $v=0-5 . K$ is the number of basis functions. 
TABLE II. Transition frequencies for $\mathrm{LiH}^{+}$vibrational states obtained in this work with $3200,3600,4000$, $4400,4800,5200$, and 5600 function basis for each state vs the frequencies of Berriche and Gadea (Ref. 5). All values are in $\mathrm{cm}^{-1}$.

\begin{tabular}{lrrrrrrrr}
\hline \hline$v^{\prime} \rightarrow v$ & 3200 & 3600 & 4000 & 4400 & 4800 & 5200 & 5600 & Berriche and Gadea \\
\hline $1 \rightarrow 0$ & 355.411 & 355.338 & 355.279 & 355.241 & 355.197 & 355.158 & 355.125 & 357.43 \\
$2 \rightarrow 1$ & 262.027 & 261.901 & 261.828 & 261.775 & 261.757 & 261.754 & 261.757 & 265.61 \\
$3 \rightarrow 2$ & 170.592 & 170.364 & 170.240 & 170.160 & 170.107 & 170.070 & 170.047 & 172.96 \\
$4 \rightarrow 3$ & 90.480 & 90.176 & 90.018 & 89.945 & 89.911 & 89.889 & 89.874 & 92.20 \\
$5 \rightarrow 4$ & 44.990 & 37.691 & 36.121 & 35.647 & 35.460 & 35.367 & 35.331 & 37.45 \\
$6 \rightarrow 5$ & & & & & & & & 9.22 \\
\hline \hline
\end{tabular}

$\mathrm{Li}\left({ }^{7} \mathrm{Li}\right.$ isotop) and $\mathrm{H}$ nuclei used in the calculations were $m_{\mathrm{Li}}=12786.3933 m_{e}$ and $m_{p}=1836.15267261 m_{e}$, where $m_{e}$ stands for the mass of the electron.

\section{RESULTS AND DISCUSSION}

The previous calculations of Berriche and Gadea ${ }^{5}$ of the vibrational spectrum in the ground electronic state of the $\mathrm{LiH}^{+}$system predicted the existence of seven bound vibrational states. Those calculations were done within the BornOppenheimer approximations and the electronic energy was determined with a method that utilized an effective core potential. Thus, they can be expected to be significantly less accurate than the calculations presented in this work. We start the presentation of the results by showing the convergence of the total energies of the ground and excited vibrational states as the function of the size of the basis set. The number of states whose energies we have managed to converge below the dissociation threshold was 6 . An attempt made to converge the seventh state below the dissociation threshold failed. With nearly 5000 functions in the basis set the energy was still above the threshold and the convergence pattern did not indicate a realistic possibility of lowering the energy below the threshold. The convergence patterns of the energies of the six vibrational states are shown in Table I. They are also shown on Fig. 1 where, for each state, we plotted the difference between the energy obtained with a particular number of basis functions and the energy obtained with 5600 functions. As mentioned, it takes more basis functions to obtain the same quality of the results for higher excited states than for the lower states because of the larger number of radial nodes in the wave functions of the higher states. Figure 1 demonstrates this trend very well. The curve corresponding to the ground state $(v=0)$ is flat, indicating that the energy of this state is already very well converged with 4500 basis functions while the energy of $v=5$ state with that many functions is still far from the same degree of convergence.

In the case of slightly lower accuracy of the calculated energy value for the $v+1$ state than for the $v$ state the transition energies, $E_{v+1}-E_{v}$, are usually slightly overestimated. Thus, our calculated transition energies shown in Table II should provide upper bounds to the exact results. In Table II we not only include the transition energies obtained with the largest basis set of 5600 functions but we also show how the energies converge with the number of basis functions. The convergence pattern allows us to estimate the accuracy of our calculations. It is safe to state that this accuracy is of the order of $0.1 \mathrm{~cm}^{-1}$. Further extension of the basis set size would certainly lead to higher accuracy. However, at this point, after over six months of continuous calculations, we have reached the limits of our computer capabilities.

In Table II we also compare our transition energies with those of Berriche and Gadea $^{5}$ All their energies are higher than ours by $2-4 \mathrm{~cm}^{-1}$. To what extent this is an effect of not assuming the Born-Oppenheimer approximation in our calculations and/or to what extent this results from inaccuracies of the potential energy curve calculated by Berriche and Gadea, it is, at this point, difficult to say.

Finally, in Table III we present the expectation values of the interparticle distances and their squares for the six bound vibrational states obtained with 5600 basis functions for each state. Those include lithium nucleus-proton $\left(r_{\mathrm{Li}-p}\right)$, lithium nucleus-electron $\left(r_{\mathrm{Li}-e}\right)$, proton-electron $\left(r_{p-e}\right)$, and electronelectron $\left(r_{e-e}\right)$ distances. The results show that the internuclear distance increases, as expected, with the vibrational excitation. It also can be noticed that the average value of the proton-electron distance parallels the average value of the electron-electron distance. This indicates that when the vibrational excitation moves the nuclei further apart, two elec-

TABLE III. Mean interparticle distances of $\mathrm{LiH}^{+}$and mean squares of the interparticle distances calculated with 5600 basis functions for each state. Here, $r_{\mathrm{Li}-p} \equiv r_{1}, r_{\mathrm{Li}-e} \equiv r_{2}, r_{p-e} \equiv r_{12}$, and $r_{e-e} \equiv r_{23}$. All values are in a.u.

\begin{tabular}{lcccccccc}
\hline \hline$v$ & $\left\langle r_{\mathrm{Li}-p}\right\rangle$ & $\left\langle r_{\mathrm{Li}-e}\right\rangle$ & $\left\langle r_{p-e}\right\rangle$ & $\left\langle r_{\mathrm{e}-\mathrm{e}}\right\rangle$ & $\left\langle r_{\mathrm{Li}-p}^{2}\right\rangle$ & $\left\langle r_{\mathrm{Li}-e}^{2}\right\rangle$ & $\left\langle r_{p-e}^{2}\right\rangle$ & $\left\langle r_{e-e}^{2}\right\rangle$ \\
\hline 0 & 4.32922 & 1.82963 & 3.41524 & 3.20863 & 18.9229 & 6.96233 & 13.9343 & 13.9400 \\
1 & 4.82188 & 1.99562 & 3.74209 & 3.53807 & 23.8518 & 8.63460 & 17.2254 & 17.2837 \\
2 & 5.53666 & 2.23690 & 4.21553 & 4.01766 & 31.8581 & 11.3554 & 22.5621 & 22.7244 \\
3 & 6.68100 & 2.62209 & 4.97388 & 4.78455 & 46.7935 & 16.4140 & 32.5126 & 32.8408 \\
4 & 8.70693 & 3.30029 & 6.31915 & 6.13698 & 79.7911 & 27.5190 & 54.5023 & 55.0501 \\
5 & 12.7210 & 4.63819 & 8.99011 & 8.80871 & 170.743 & 57.9530 & 115.130 & 115.918 \\
\hline \hline
\end{tabular}



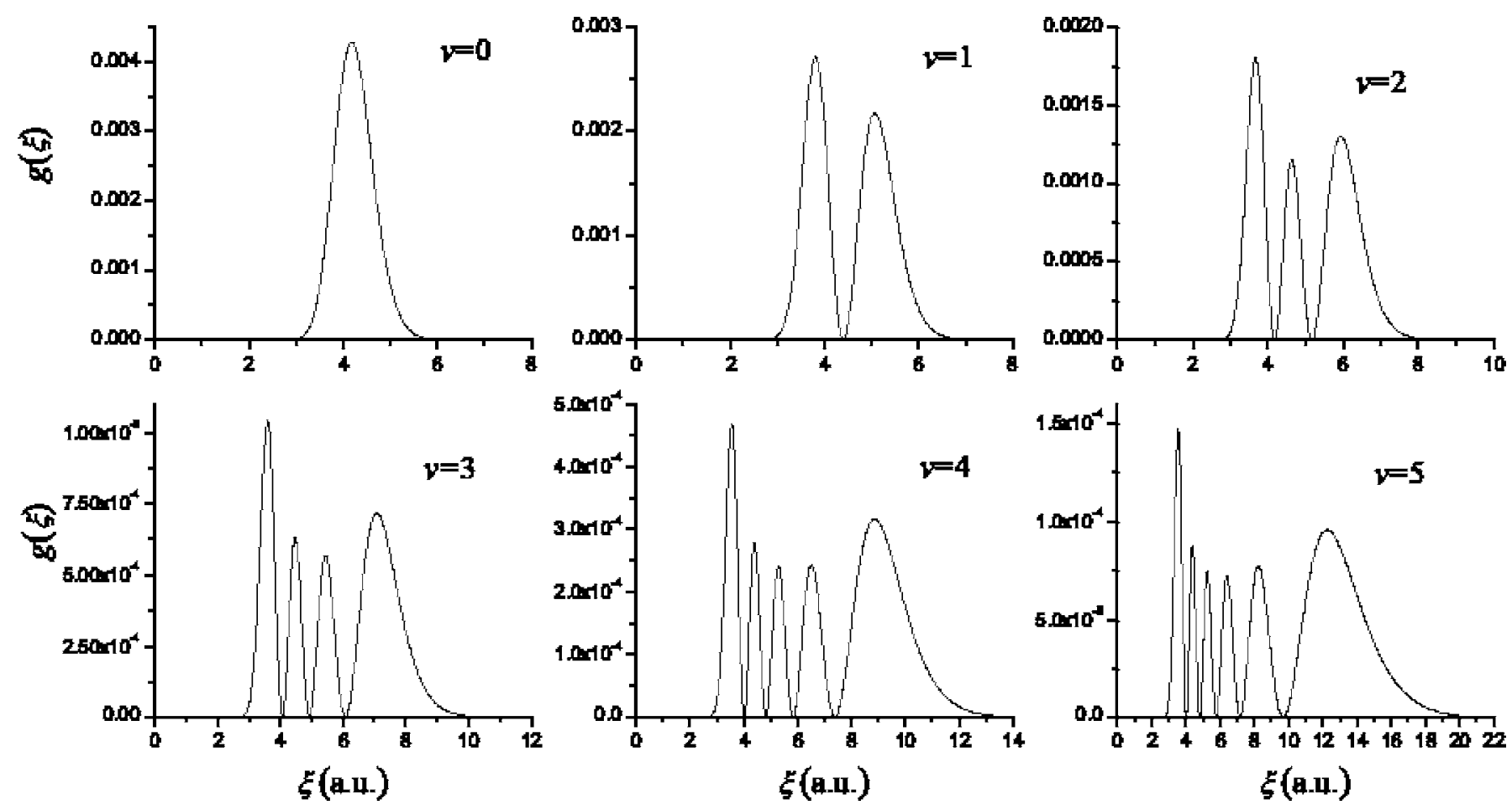

FIG. 2. Nucleus-nucleus correlation function for $v=0-5$ vibrational states of $\mathrm{LiH}^{+}$.

trons stay with the Li nucleus and one moves away with the proton. Thus, at the limit $\mathrm{LiH}^{+}$dissociates into $\mathrm{Li}^{+}$and $\mathrm{H}$.

The increase of the internuclear distance with the vibrational excitation is the result of the radial function describing the system becoming increasingly more diffuse and oscillating more. This effect is shown in Fig. 2 where the nucleusnucleus correlation function, which is the same as onepseudoparticle density corresponding to pseudoparticle 1 (i.e., pseudoproton), is plotted for all vibrational states we calculated in this work. This function is defined as (see Ref. 10)

$$
g(\boldsymbol{\xi})=\left\langle\Psi(\mathbf{r})\left|\delta\left(\mathbf{r}_{1}-\boldsymbol{\xi}\right)\right| \Psi(\mathbf{r})\right\rangle,
$$

where $\Psi(\mathbf{r})$ is the total wave function of the system under consideration.

\section{SUMMARY}

In this work we performed very accurate non-BO calculations on fully symmetric bound states of the $\mathrm{LiH}^{+}$ion and we found six such states. These states are conventionally called vibrational states although, if the $\mathrm{BO}$ approximation is not assumed, the vibrational quantum number is, strictly speaking, not a good quantum number due to the coupling between the electronic and vibrational motions. We estimated that the accuracy of our vibrational transition energies calculated as differences between the energies of adjacent energy levels is about $0.1 \mathrm{~cm}^{-1}$ in relation to the exact transitions (calculated without including relativistic corrections). This makes, to our knowledge, the present calculations the most accurate ever performed for a diatomic system with three electrons.

\section{ACKNOWLEDGMENTS}

This work has been supported in part by the National Science Foundation. We would also like to thank University of Arizona Center of Computing and Information Technology for the computer time on HP Alpha GS1280 parallel computer system.

\footnotetext{
${ }^{1}$ S. Bubin and L. Adamowicz, J. Chem. Phys. 118, 3079 (2003).

${ }^{2}$ M. Stanke, D. Kẹdziera, S. Bubin, M. Barysz, and L. Adamowicz, J. Chem. Phys. 125, 014318 (2006).

${ }^{3}$ L. Wolniewicz, J. Chem. Phys. 99, 1851 (1993).

${ }^{4}$ O. Grabandt, H. J. Bakker, and C. A. de Langes, Chem. Phys. Lett. 189, 291 (1992).

${ }^{5}$ H. Berriche and F. X. Gadea, Chem. Phys. 203, 373 (1996).

${ }^{6}$ M. Cafiero, S. Bubin, and L. Adamowicz, Phys. Chem. Chem. Phys. 5, $1491(2003)$

${ }^{7}$ S. Bubin, M. Cafiero, and L. Adamowicz, Adv. Chem. Phys. 131, 377 (2005).

${ }^{8}$ S. Bubin, L. Adamowicz, and M. Molski, J. Chem. Phys. 123, 134310 (2005).

${ }^{9}$ M. Hamermesh, Group Theory and Its Application to Physical Problems (Dover, New York, 1962)

${ }^{10}$ S. Bubin and L. Adamowicz, Chem. Phys. Lett. 403, 185 (2005).
} 